\title{
Transient Stability Analysis of the SeCRETS Experiment in SULTAN
}

\author{
Claudio Marinucci, Luca Bottura, and Pierluigi Bruzzone
}

\begin{abstract}
We present here the results of the analysis of the stability experiment SeCRETS, performed on two $\mathrm{Nb}_{3} \mathrm{Sn}$ cable-inconduit conductors with the same amount of total copper stabilizer, but different degree of segregation. The model used for the analysis, including superconducting strands, conductor jacket and helium, is solved with the code Gandalf ${ }^{\mathrm{TM}}$. We obtain a qualitative agreement of simulation results and experimental values. The simulation results confirm that in the operation regime explored in the experiment the segregated copper is not effective for stability. The details of the current sharing and the approximation taken for the transient heat transfer are shown to be critical for the interpretation.
\end{abstract}

Index Terms-Cable-in-conduit conductor, current sharing, heat transfer, transient stability.

\section{INTRODUCTION}

$\mathbf{T}$ HE SEGREGATED Copper Ratio Experiment on Transient Stability (SeCRETS) has been successfully completed in the SULTAN facility at CRPP. The main aim of the experiment was to compare the stability performance of two $\mathrm{Nb}_{3} \mathrm{Sn}$ cable-in-conduit conductors (CICC) prepared with identical cross sections but different location of the copper stabilizer, i.e., either fully included in the composite strands (conductor A) or partially segregated as pure copper wires accounting for $34 \%$ of the total stabilizer in the cable (conductor B). Two conductor sections, each about $13 \mathrm{~m}$ long, were series connected and wound as a bifilar single layer solenoid. A set of pulsed coils provided a transverse field to both conductors over $30 \mathrm{~cm}$ length. After heat treatment, the instrumented winding was inserted in the bore of SULTAN with background field $B_{S U L}$ up to $11.3 \mathrm{~T}$ and operating current $I_{o p}$ up to $12 \mathrm{kA} \mathrm{[1],}$ [2].

The stability test under pulsed field was the key test for SeCRETS. After setting $B_{S U L}$ and $I_{O p}$, the temperature was adjusted to obtain in both conductors the same temperature margin, $\Delta T=T_{c s}-T_{o p}$, where $T_{c s}$ is the current sharing temperature and $T_{o p}$ the operating temperature. The pulsed coils were subsequently fired, producing a sinusoidal field variation with amplitude $\Delta B$ and period $65 \mathrm{~ms}$. The amplitude of the field waveform was increased till one of the two conductor lengths quenched. The margin $\Delta T$ was then increased in the weaker conductor to allow further testing of the stronger conductor. These tests were repeated for several settings of

Manuscript received September 24, 2001.

C. Marinucci and P. Bruzzone are with CRPP, Fusion Technology Div., $\mathrm{CH}-5232$ Villigen PSI, Switzerland.

L. Bottura is with CERN, CH-1211 Geneva 23, Switzerland (e-mail: Luca.Bottura@cern.ch).

Publisher Item Identifier S 1051-8223(02)04292-6.
TABLE I

REFERENCE INPUT DATA

\begin{tabular}{|c|c|c|c|}
\hline \multirow[t]{2}{*}{ Parameter } & \multirow[t]{2}{*}{ Units } & \multicolumn{2}{|c|}{ Conductor } \\
\hline & & A & B \\
\hline length & (m) & 2 & 2 \\
\hline $\begin{array}{l}\text { non-copper cross } \\
\text { section }\end{array}$ & $\left(\mathrm{mm}^{2}\right)$ & 30.24 & 29.89 \\
\hline copper cross section & $\left(\mathrm{mm}^{2}\right)$ & 45.36 & $29.89^{(1)}$ \\
\hline $\begin{array}{l}\text { cosine of cabling } \\
\text { angle }\end{array}$ & $(-)$ & 0.9840 & 0.9893 \\
\hline jacket cross section & $\left(\mathrm{mm}^{2}\right)$ & 46.37 & 46.37 \\
\hline helium cross section & $\left(\mathrm{mm}^{2}\right)$ & 44.07 & 44.51 \\
\hline void fraction & $(\%)$ & 36.8 & 37.2 \\
\hline $\begin{array}{l}\text { cable } \\
\text { perimeter }\end{array}$ & $(\mathrm{mm})$ & 373 & 292 \\
\hline $\begin{array}{l}\text { jacket wetted } \\
\text { perimeter }\end{array}$ & $(\mathrm{mm})$ & 9.7 & 9.7 \\
\hline hydraulic diameter & $(\mathrm{mm})$ & 0.473 & 0.512 \\
\hline longitudinal strain & $(\%)$ & -0.725 & -0.635 \\
\hline $\mathrm{T}_{\mathrm{cOM}}{ }^{(2)}$ & $(\mathrm{K})$ & 17.3 & 17.7 \\
\hline $\mathrm{B}_{\mathrm{c} 20 \mathrm{M}}{ }^{(2)}$ & $(\mathrm{T})$ & 32.65 & 32.80 \\
\hline $\mathrm{C}_{0}^{(2)}$ & $\left(\mathrm{AT}^{0.5} / \mathrm{mm}^{2}\right.$ & 7750 & 7515 \\
\hline copper RRR & $\begin{array}{l}) \\
(-) \\
\end{array}$ & 107 & 95 \\
\hline
\end{tabular}

NOTES:

(1) segregated copper not included

(2) fit parameters for $\mathrm{Nb}_{3} \mathrm{Sn}$ critical properties [7]

operating current, operating temperature and helium massflow. Details on the experiment and the main results can be found in [2], [3]. In this paper we analyze the main results focusing on the dependence of transient stability on operating conditions such as current, temperature margin and helium massflow.

\section{Simulation Model}

We have performed the simulation of the SeCRETS stability test with Gandalf ${ }^{\mathrm{TM}}$, the CryoSoft code for the simulation of quench initiation and propagation in CICC with multiple cooling channels. The model at the basis of the code and the solution method are described in [4] and [5]. The components, i.e., strands, jacket and helium, are thermally coupled through convection on wetted surfaces (or contact perimeters) with a surface heat transfer coefficient $h$. The temperature is homogeneous in the cross section of the components and can vary only along the length. Standard models were used for the heat transfer and friction factor correlations. The code, as discussed later, was however modified substantially to take into 
proper account the voltage-current relation of the cable in the calculation of the current sharing and Joule heat dissipation.

\section{A. Cable Geometry and Operating Conditions}

The reference input data is summarized in Table I. The length of conductor modeled is $2 \mathrm{~m}$ and its center coincides with the center of the heated zone. We have verified that the transient stability is decided shortly after the end of the field pulse, hence the boundary conditions at the end of the sample do not affect the result. It is therefore not necessary to model the full length of $13 \mathrm{~m}$. Measured values of the helium inlet pressure and of the massflow are given as hydraulic boundary conditions. In both conductors the copper and noncopper cross sections used include a $1 / \cos (\theta)$ correction due to conductor twisting by a cabling angle $\theta$. While in conductor A the full copper cross section is considered, in conductor B the segregated copper cross section is neglected. Taking this choice we make the drastic assumptions that only the copper embedded in the strands participates to the current sharing. For this reason only the wetted perimeter of the strands is accounted for the heat transfer to helium. The heat transfer perimeter at the contact surface of strand and bundle is the geometric value, i.e., the sum of the wetted perimeters of the strands. The friction factor of the helium flow in the cable bundle is characterized by the correlation of Katheder [6]. The mesh consists of 200 linear elements and the minimum and maximum time steps used for integration are $1 \mu \mathrm{s}$ and $10 \mu \mathrm{s}$, respectively. A first order method in time is applied for the integration. We checked that the simulations are numerically converged for this choice of solution parameters.

\section{B. Pulsed Magnetic Field and Deposited Energy}

In the experiment the energy is deposited in the cable by the AC loss caused by the sinus variation of the pulsed field, superimposed to the constant background field $B_{S U L}$. In the simulation we have considered the combined effect of the simultaneous field variation, affecting the critical properties of the superconductor, and of the energy deposition. We have taken for the pulsed field the following waveform:

$$
B=\Delta B \sin \left(2 \pi \frac{t}{\tau_{q}}\right)
$$

where $\tau_{q}=65 \mathrm{~ms}$ is the period of the pulse and $\Delta B$ its amplitude. We further assumed that during this field pulse the AC loss is in fully developed (resistive) regime, so that the time dependence of the power dissipated $Q$ is proportional to the square of the time derivative of the field $d B / d t$, or:

$$
Q=Q_{0}\left[\cos \left(2 \pi \frac{t}{\tau_{q}}\right)\right]^{2}
$$

where $Q_{0}$ is a proportionality constant adjusted during the iterative search of the stability margin. In the model the heat is deposited uniformly in the cross section of the strands, and is constant along the length of the pulsed coils, i.e., between $0.85 \mathrm{~m}$ and $1.15 \mathrm{~m}$.
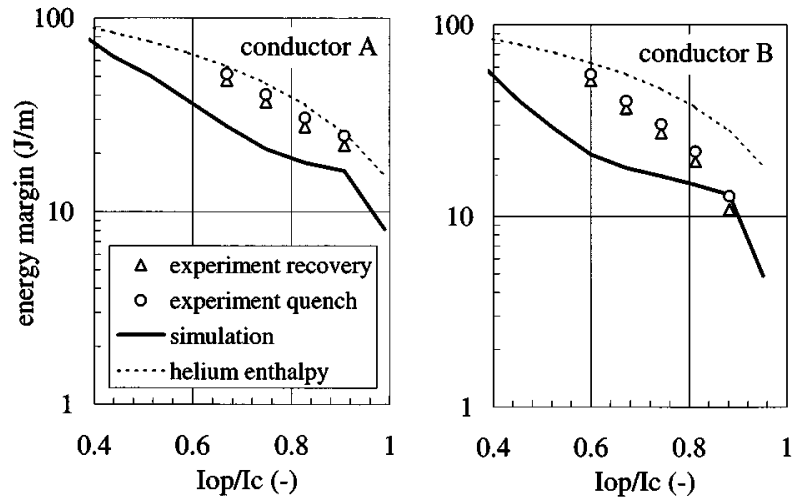

Fig. 1. Transient stability as a function of the ratio of operating to critical current for conductor A (left) and B (right) at a massflow of $3.5 \mathrm{~g} / \mathrm{s}$. The operating temperature is $6.2 \mathrm{~K}$ for conductor $\mathrm{A}$ and $6.6 \mathrm{~K}$ for $\mathrm{B}$. The experimental results (symbols) are compared to the results of simulations (solid line). The helium enthalpy between the operating temperature and the current sharing temperature represents the ideal upper limit of the energy margin (dotted line).

\section{Superconductor Properties}

The critical current density $J_{c}$ is fit using [7] and the coefficients of Table I. It was found in the experiment that the value of $T_{c s}$ depends strongly on the value of the electric field $E_{0}$ at which the critical current is defined. The reason is that with a power-law exponent $n$ of the order of 15 , the voltage rise in the proximity of the critical current is relatively shallow and a considerable voltage can be sustained before thermal runaway. The difference in the $T_{c s}$ deduced at $1 \mu \mathrm{V} / \mathrm{cm}$ and the temperature at which the thermal runaway takes place can be as large as $0.6 \mathrm{~K}$. As most of the experimental runs were performed in a region where the temperature margin was below $1 \mathrm{~K}$, this effect can be very important and had to be included in the model.

The model originally devised in Gandalf ${ }^{\mathrm{TM}}$ for the current sharing is equivalent to a value of $n$ equal to infinity [5]. This results in a large underestimate of the stability margin. The calculation of the Joule heat contribution was thus modified to model the effect of a finite $n$. To this aim the electric field in the superconductor was modeled as is customary using a power law approximation:

$$
E=E_{0}\left(\frac{I_{s c}}{I_{c}}\right)^{n}
$$

where $E_{0}$ is the electrical field used for the definition of the critical current $I_{c}, I_{s c}$ is the current in the superconductor (i.e., excluding the current shared by the stabilizer) and $n$ is the exponent of the power law. The values taken in the simulations are $E_{0}=1 \mu \mathrm{V} / \mathrm{cm}$ and $n=15$. The total Joule power density $Q_{J o u l e}$ (in $\mathrm{W} / \mathrm{m}$ ) is then obtained as the scalar product of current and electric field:

$$
Q_{\text {Joule }}=E I .
$$

\section{RESULTS AND DISCUSSION}

As a measure of transient stability we have used the energy margin per unit conductor length $\Delta E(\mathrm{~J} / \mathrm{m})$. This is defined in 


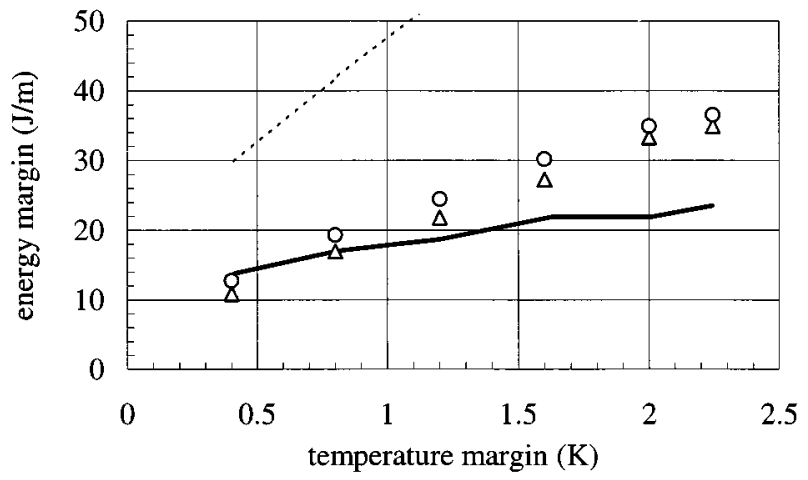

Fig. 2. Measured (symbols) and computed (solid line) transient stability of conductor $\mathrm{B}$ as a function of the temperature margin, for operation at $12 \mathrm{kA}$ current and $3.5 \mathrm{~g} / \mathrm{s}$ massflow.

the simulations as the integral of the power in (2), averaged between the highest value that leads to a recovery and the lowest value resulting in a thermal runaway. The experimental values used for comparison are obtained by energy calibration of the amplitudes of the two field pulses right before the quench value and producing the quench. The energy calibration was obtained using gas-flow calorimetry and is described elsewhere [3]. For comparison, we have also reported in all figures the difference of helium enthalpy between $T_{o p}$ and $T_{c s}$ which represents an ideal upper bound for the heat absorption capability of the conductor in case of infinite heat exchange between strands and helium. All results reported here refer to the runs with background field $B_{S U L}=9.71 \mathrm{~T}$.

\section{A. Stability as a Function of the Operating Current}

The comparison of measured and computed transient stability as a function of the operating current fraction is shown in Fig. 1 for conductors $\mathrm{A}$ and $\mathrm{B}$ respectively. The operating temperature is constant at $6.2 \mathrm{~K}$ in conductor $\mathrm{A}$ and $6.6 \mathrm{~K}$ in conductor $\mathrm{B}$. The massflow for both conductors is $3.5 \mathrm{~g} / \mathrm{s}$. The simulation is performed in a range $I_{o p} / I_{C}$ from 0.4 to 1 , i.e., broader than the experimental range. Taking an average value of the heat transfer coefficient of $1000 \mathrm{~W} / \mathrm{m}^{2} \mathrm{~K}$ the expected limiting current fraction for conductor A is at $I_{l i m} / I_{c} \approx 0.9$, and $I_{l i m} / I_{c} \approx 0.6$ for conductor B [8].

For conductor A both simulation and experiment do not show any evident drop, as expected because of the high limiting current fraction. The difference between experimental data and simulation is approximately $40 \%$ in the full range of currents. For conductor B the simulation shows a mild drop of the stability margin around the expected limiting current, below $I_{o p} / I_{c} \approx 0.6$, not visible in the experimental measurements. In this case the difference between experiment and simulation is less than 5\% at high current, but grows to as much as $60 \%$ at low current. In general, the simulated energy margin tends to under-estimate the measured values, in particular at low $I_{o p}$. We attribute this result to the pessimistic assumptions taken for the transient heat transfer, as discussed later. We finally note that the simulation reproduces the experimental result in that the computed stability of conductor A (with copper fully distributed among the superconducting strands) is superior to conductor B (with segregated copper).

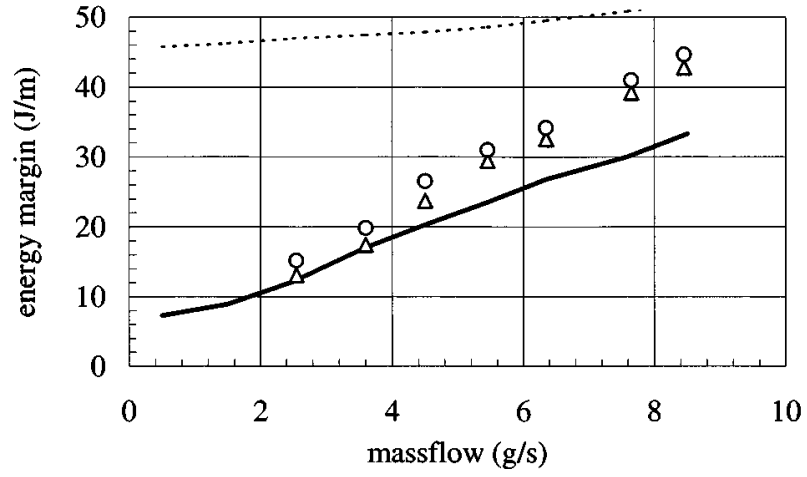

Fig. 3. Measured (symbols) and computed (solid line) transient stability of conductor B as a function of the helium massflow, for operation at $12 \mathrm{kA}$ current $1 \mathrm{~K}$ temperature margin.

\section{B. Stability as a Function of the Temperature Margin}

The effect of the temperature margin $\Delta T$ was investigated in the range $0.4 \mathrm{~K}-2.3 \mathrm{~K}$ with operating current $12 \mathrm{KA}$ and massflow $3.5 \mathrm{~g} / \mathrm{s}$. We show in Fig. 2 the results for conductor B. The simulation agrees qualitatively with the experimental results that show a linear dependence of the energy margin on the temperature margin. In this regime of operation (high operating current fraction), the measured and computed stability margin is significantly smaller than the helium enthalpy for the corresponding temperature margin. The difference between simulated and experimental $\Delta E$ is small (less than $5 \%$ ) up to $\Delta T=$ $1 \mathrm{~K}$. At higher $\Delta T \mathrm{~s}$ the simulation underestimates the measured energy margin, with a difference of $36 \%$ at $\Delta T=2.3 \mathrm{~K}$. The results of conductor A are similar but show a constant offset of approximately $10 \mathrm{~J} / \mathrm{m}$. Note finally that without taking into account the smooth electric field transition, i.e., with an infinite value for $n$, the simulation results would be affected by a $0.5 \mathrm{~K}$ shift that would lead to a clear disagreement with the experimental results.

\section{Stability as a Function of the Helium Massflow}

The effect of helium massflow is investigated operating at a current of $12 \mathrm{kA}$ with a temperature margin of $1 \mathrm{~K}$ in both conductors, i.e., at an operating temperature of $5.53 \mathrm{~K}$ in conductor $\mathrm{A}$ and $5.95 \mathrm{~K}$ in conductor $\mathrm{B}$. The comparison of experimental and computer results is shown in Fig. 3. The simulation broadly agrees with the experimental results, showing a linear dependence of energy margin on massflow. The difference is only few percent below $4 \mathrm{~g} / \mathrm{s}$ and reaches $25 \%$ at $8 \mathrm{~g} / \mathrm{s}$. The results for conductor A show a slightly better agreement.

To explain the linear dependence we recall that higher massflow results in higher turbulence and thus an enhancement of the steady state heat transfer coefficient. The linear dependence of $\Delta E$ on massflow points to the fact that the stability in both conductors is limited by heat transfer, as discussed next.

\section{Discussion}

The results presented show that the experimental energy margin exceeds, sometimes significantly, the expected value, approaching the theoretical upper limit given by the helium enthalpy between operating and current sharing temperature, as would be typical for operation in the well-cooled regime 


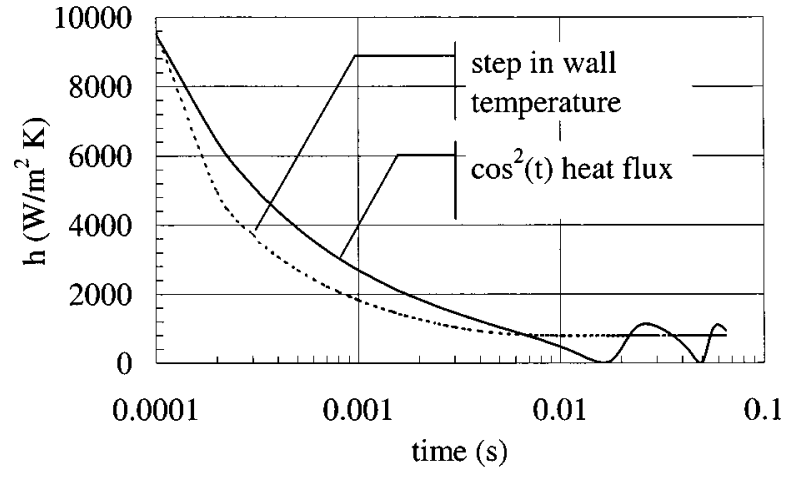

Fig. 4. Simulation of heat transfer in an ideal boundary layer with $25 \mu \mathrm{m}$ thickness and constant thermophysical properties. The equivalent heat transfer coefficient for the boundary layer is computed analytically for a step in the strand temperature (the approximation used in Gandalf) and numerically for a time variable heat flux from the strand with a $\cos ^{2}(t)$ dependence.

[8]. In addition the stability plots as a function of operating current fraction does not show a limiting current. On the other hand, as shown by the massflow dependence in Fig. 3, we have evidence that the stability is limited by heat transfer, as typical of the ill-cooled regime [8]. This apparent contradiction can be resolved considering on one hand the relatively long time of energy deposition, and on the other hand the restricted operating regime explored, limited to small temperature margin. The energy that can be transferred to the helium bath during the field pulse, proportional to the pulse duration, can be significant when compared to the available helium enthalpy, proportional to the temperature margin. This phenomenon results in the large energy margin, compared to the helium enthalpy, even in the ill-cooled regime, and is qualitatively reproduced by the simulation.

As anticipated, we attribute the main differences between experiment and simulation to the approximation used for the transient heat transfer coefficient $h$. This approximation is based on the analytical solution of the heat flux entering an ideally infinite boundary layer following a step in the strand temperature [9]. In reality, during the experiment the heat flux from the strand to the helium is a strong function of time, and thus the assumption of constant strand temperature used for the calculation of $h$ fails. To demonstrate the influence of this effect we have performed a simulation of the temperature evolution in a helium boundary layer with constant thermophysical properties and constant thickness of $25 \mu \mathrm{m}$. One side of the boundary layer is bounded by the strand surface while the other side is kept at the bulk helium temperature. The equivalent heat transfer coefficient for a step in the strand temperature is compared in Fig. 4 to the heat transfer coefficient obtained for a $\cos ^{2}(t)$ variation of the heat flux at the strand surface, simulating the heat deposition in the experiment. The difference is striking, in spite of the simplistic approach taken, and demonstrates that the approxi- mation used may be quite far from reality. In particular the transient heat transfer can be significantly larger than what we have assumed, especially at the end of the heat pulse, when recovery takes place. Variable thermophysical properties, turbulence, the resulting changes in boundary layer thickness, and a complex geometry make a more complete analysis impractical.

\section{CONCLUSIONS}

We have performed a numerical analysis of the transient stability experiment SeCRETS on two $\mathrm{Nb}_{3} \mathrm{Sn}$ cable-in-conduit conductors. The numerical study of the experimental results has revealed details on the fact that the stability in the operating regime explored (low temperature margin in proximity of the normal transition) is limited by heat transfer. The stability of both samples is excellent, although the segregated stabilizer does not participate to the current sharing process. The adverse effect of copper segregation on stability is qualitatively reproduced by the simulations.

We have found that a consistent model of the voltage-current characteristic of the conductor is required to obtain accurate predictions of stability. In the case of the SeCRETS conductor the use of the power-law model described has resulted in an increase of the effective temperature margin by approximately $0.5 \mathrm{~K}$. For design and scoping calculations the typical accuracy that can be achieved with the model described here is appropriate. We remark however that a better knowledge of the details of transient heat transfer is necessary for more accurate prediction of stability.

\section{REFERENCES}

[1] P. Bruzzone, "Segregated copper ratio experiment on transient stability (SeCRETS) final report,", CRPP Report LRP 689/01, ISSN 0458-5895, Jan. 2001.

[2] P. Bruzzone et al., "SeCRETS: A atability experiment on the role of segregated copper in $\mathrm{Nb}_{3} \mathrm{Sn}$ cable-in-conduit conductors," IEEE Trans. Appl. Superconduct., vol. 10, no. 1, pp. 1082-1085, 2000.

[3] P. Bruzzone, A. Fuchs, B. Stepanov, G. Vecsey, and E. Zepretilina, "Test results of SeCRETS, a stability experiment about segregated copper in CICC," IEEE Trans. Appl. Superconduct., vol. 11, no. 1, pp. 2018-2021, 2001.

[4] L. Bottura, "Modeling stability in superconducting cables," Phys. C, vol. 310, pp. 316-326, 1998.

[5] L. Bottura, "A numerical model for the simulation of quench in the ITER magnets," J. Comp. Phys., vol. 125, pp. 26-41, 1996.

[6] H. Katheder, "Optimum thermohydraulic operation regime for cable in conduit superconductors (CICS)," Cryogenics, vol. 34, pp. 595-598, 1994.

[7] L. T. Summers, M. W. Guinan, J. R. Miller, and P. A. Hahn, "A model for the prediction of $\mathrm{Nb}_{3} \mathrm{Sn}$ critical current as a function of field, temperature, strain, and radiation damage," IEEE Trans. Magn., vol. 27, no. 2, pp. 2041-2044, 1991.

[8] L. Bottura, "Cable in conduits," in Handbook of Applied Superconductivity, B. Seeber, Ed. Bristol: IOP Publishing, 1998, ch. B.4.

[9] W. B. Bloem, "Transient heat transfer to a forced flow of supercritical helium at 4.2 K," Cryogenics, vol. 26, pp. 300-308, 1986. 